\title{
MORTALIDAD MATERNA EN LA GESTANTE WAYÚU DE URIBIA, DEPARTAMENTO DE LA GUAJIRA, COLOMBIA. ESTUDIO DESCRIPTIVO AÑO 2016
}

\author{
MATERNAL MORTALITY IN THE PREGNANT WAYÚU WOMEN OF URIBIA, \\ DEPARTMENT OF LA GUAJIRA, COLOMBIA. DESCRIPTIVE STUDY 2016 \\ A MORTALIDADE MATERNA NA GRÁVIDA WAYUU DE URIBIA, \\ DEPARTAMENTO NA GUAJIRA, COLÔMBIA. ESTUDO DESCRIPTIVO 2016
}

\author{
Javier Alonso Bula Romero \\ Universidad Antonio Nariño. Bogotá, Colombia. \\ Jabular@unal.edu.co
}

ORCID: 0000-0002-0788-0472

Keiner Galarza

Universidad Antonio Nariño. Bogotá, Colombia.

ORCID: 0000-0002-2744-4491 http://dx.doi.org/10.22235/ech.v6i1.1369

Recibido: $28 / 02 / 2017$

Aceptado: 22/03/2017

\section{RESUMEN}

La tendencia de mortalidad materna en el municipio de Uribia, Departamento de la Guajira, Colombia, ha mostrado un ascenso vertiginoso. Si bien existen esfuerzos por mejorar la cobertura en la atención del parto institucional en el municipio, el parto domiciliario, que es una práctica cultural habitual de poblaciones indígenas como la Wayúu, sigue siendo, por diversas razones, la primera opción para un número significativo de mujeres Wayúu. Esta práctica cultural expone a las mujeres a complicaciones durante la atención del parto que, de no ser remitidas a tiempo a un centro hospitalario, conducen a una muerte materna. La presente investigación tuvo como objetivo describir el comportamiento de la mortalidad materna en la gestante Wayúu del municipio de Uribia en el periodo comprendido entre el primero de enero del 2010 al 31 de diciembre del 2016. Los resultados de este estudio muestran cómo las prácticas tradicionales de parto, el difícil acceso a los servicios de salud y la edad de la madre son factores que contribuyen a aumentar la tasa de mortalidad materna y esto, a su vez, revela la vulnerabilidad social y de salud a la que está expuesta esta comunidad indígena. La investigación concluyó además que la hemorragia posparto fue la principal causa de mortalidad materna en la gestante Wayuu del municipio de Uribia, durante el período estudiado. La atención domiciliaria del parto, realizada por madres, abuelas o hermanas mayores, puede ser un factor desencadenante de este tipo de complicaciones obstétricas que, si no son atendidas adecuadamente, pueden conducir a la muerte de la madre.

Palabras clave: Mortalidad Materna, Mujeres Embarazas, Características Culturales, Salud de Poblaciones Indígenas.

\section{ABSTRACT}

The trend of maternal mortality rate in the municipality of Uribia, Department of Guajira, Colombia, has shown a dizzying increase. Although there are efforts to improve the coverage of childbirth occurring in institutions of the municipality, home deliveries, which are a normal cultural practice in indigenous populations such as the Wayúu, remains, for various reasons, the first option for a significant number of Wayúu women. This cultural practice exposes women to complications during childbirth care, which, if not sent to a hospital in time, can result in maternal death. The objective of the present study was to describe the behavior of maternal mortality in the pregnant Wayúu of the municipality of Uribia in the period between January 1st, 2010 and December 31, 2016. The results of this study show how traditional practices of childbirth, the difficulty of access to health services and the mother's age are factors that contribute to increase the maternal mortality rate and this, in turn, reveals the social and health vulnerability to which this indigenous community is exposed. The study also concluded that postpartum hemorrhage (PPH) was the main cause of maternal mortality in the pregnant woman Wayuu of the municipality of Uribia, during the period studied. Home care at the delivery, performed by mothers, grandmothers or older sisters, can be a trigger for this type of obstetric complications that, if not addressed properly, can lead to the death of the mother.

Keywords: Maternal Mortality, Pregnant Women, Cultural Characteristics, Health of Indigenous Peoples. 


\section{RESUMO}

A tendência da mortalidade materna no município de Uribia, Departamento de Guajira, Colômbia, mostrou um rápido aumento. Embora existam esforços para melhorar a cobertura de cuidados ao parto institucional no município, o parto em domicílio, que é uma prática cultural comum dos povos indígenas como o Wayuu, permanece, por várias razões, a primeira escolha para um número significativo de mulheres Wayúu. Esta prática cultural expõe as mulheres a complicações durante a assistência ao parto que, se não oportuna ser enviado para um hospital, levam à morte materna. Esta pesquisa teve como objetivo descrever o comportamento da mortalidade materna no Wayuu gestante no município de Uribia no período de 1 de Janeiro de 2010 a 31 de Dezembro de 2016. Os resultados deste estudo mostram fazer tradicional práticas do nascimento , dificuldade de acesso aos serviços de saúde e idade materna são fatores que contribuem para o aumento da taxa de mortalidade materna e este, por sua vez, revela a vulnerabilidade social e de saúde que esta comunidade indígena está exposto. A investigação também concluiu que a hemorragia pós-parto (PPH) foi a principal causa de mortalidade materna em Wayuu gestante o concelho de Uribia, durante o período de estudo. Oparto em domicílio cuidado, pelas mães, avós e irmãs mais velhas, pode ser um gatilho para este tipo de complicações obstétricas, se não forem devidamente tratados, podem levar a fator de morte materna.

Palavras-chave: Mortalidade Materna, Gestantes, Características Culturais, Saúde de Populações Indígenas.

\section{INTRODUCCIÓN}

La salud materna no sólo es el resultado de las condiciones de las mujeres durante la gestación, el parto y el posparto, sino que es un indicador del grado de desarrollo de un país y su capacidad para garantizar derechos humanos y salud a su población (1-7). Las condiciones de desigualdad e inequidad que enfrentan las mujeres en distintas regiones del mundo se reflejan especialmente en los casos de muerte materna y representan serias implicaciones no sólo para las mujeres en gestación y sus hijos por nacer, sino para sus familias y la sociedad en general. El bajo nivel educativo de las gestantes, las barreras geográficas, económicas, culturales y de calidad de la atención en salud son elementos causales relacionados con la mortalidad materna (8).

Cada día se producen en el mundo aproximadamente 830 muertes de mujeres por causas relacionadas con el embarazo y el parto. Casi todas estas muertes suceden en países en desarrollo y, en su mayoría, podrían haber sido evitadas (9). En 2015 la razón de mortalidad materna en los países en desarrollo fue de 239 por 100000 nacidos vivos, mientras que en los países desarrollados fue tan sólo de 12 por 100000 nacidos vivos (9). En el contexto colombiano, la razón de esta mortalidad es 3,02 veces más alta en los departamentos con mayor Índice de Pobreza Multidimensional. Entre ellos se destaca el Departamento de la Guajira, que posee además la particularidad de tener uno de los principales asentamientos indígenas en el territorio nacional. El caso de las mujeres indígenas del Departamento de la Guajira representa una de las tasas más altas de mortalidad materna en Colombia: para el año 2015, esta región presentaba una Razón de Mortalidad Materna por encima de las 100 muertes por 100000 nacidos vivos (1).

El Departamento de la Guajira está localizado al norte de Colombia. Cuenta con una extensión de 20.848 kilómetros cuadrados, lo que representa el $1,8 \%$ del territorio nacional $(4,10$, 11). En este departamento se concentra el mayor número de población indígena del país, especialmente de la comunidad Wayúu $(4,11)$. Ocupando más de la tercera parte de la superficie terrestre del Departamento de la Guajira, el Municipio de Uribia se ubica en el extremo más septentrional de América del Sur y tiene una posición marítima y fronteriza estratégica en la región. Se estima que cerca del 95,9\% de la población total del municipio pertenece al pueblo Wayúu (1-4, 10-12).

A pesar de contar con una importante cobertura en los servicios de salud, hay un porcentaje significativo de la población rural del departamento que no accede ni hace uso de estos servicios por motivos culturales, geográficos e institucionales. Esta situación, entre otras prácticas culturales propias de las mujeres de esta comunidad, impacta negativamente los indicadores de morbimortalidad materno-perinatal de la población indígena Wayúu, como lo demuestran las estadísticas (13-16).

\section{Prácticas de cuidado cultural relacionadas con el embarazo, parto y posparto de la mujer Wayúu}

Desde el inicio de su pubertad la mujer Wayúu participa en distintas tradiciones de su comunidad asociadas a la sexualidad y la vida fértil (15-19). Las mujeres embarazadas toman consejos de su madre o sus hermanas mayores, en especial durante su primer embarazo, pues la preocupación por la llegada del parto está determinada por el número de hijos que la mujer haya tenido: la primeriza recibe más atención que las mujeres que han parido varias veces, pues se dice que a ellas «el niño les sale solito, solito, sin problema» $(13,15,16)$. La gestante sigue recomendaciones para protegerse a sí misma y al bebé: procura no salir de noche, ni ir al monte, al jagüey ${ }^{1}$, a velorios o entierros, con el fin de evitar los espíritus malignos que puedan afectarla a ella o a su hijo (18).

Aunque algunas mujeres jóvenes están empezando a acudir más a los hospitales, los partos son por lo general atendidos en casa por un familiar, por una partera o, en algunos casos, por la misma gestante (14-16). La nativa Wayúu, con el fin de prepararse para el parto, se lava el cuerpo y el cabello, se limpia el pubis y se coloca ropa limpia. Asimismo, alista el sitio donde tendrá lugar el parto disponiendo de una hamaca o una soga para sujetarse. La madre se acuesta o se sienta sobre un asiento de madera para llevar a cabo el parto $(15,16)$. Cuando realiza el parto sentada, la madre pone su espalda contra un chin-

1 Palabra propia de la cultura wayúu. Hace referencia al pozo de donde se obtiene el agua. 
chorro colgado y extiende sus brazos tomándose del mismo; frente a ella está ubicada una capa de arena cubierta con una sábana para colectar la sangre derramada y sacarla de la casa después (14). En caso de que el parto se complique, se llama a la partera o se transporta a la mujer al hospital si las distancias lo permiten $(15,16)$. Tras dar a luz, se lava con agua de hierbas, se le hace un masaje en el abdomen y se le dan bebidas para el dolor y para el sangrado $(15,16)$.

La placenta debe ser enterrada. Si el nacimiento ocurre en un hospital, le piden la placenta al médico para enterrarla en la ranchería (18-20). De igual manera, el ombligo del recién nacido se limpia, se amarra con hilo de coser blanco tratado con yerbas medicinales $y$, según la tradición cultural, las madres lo guardan en una cajita y posteriormente lo entierran para que los hijos permanezcan en sus territorios $(18,19)$.

La lactancia materna es la única fuente de alimentación que recibe el recién nacido; si ésta no es abundante, la madre toma chicha de maíz o leche de cabra $(15,16)$. Cuando la leche de la madre no baja, le masajean los senos después de calentarse las manos en ceniza caliente y le ofrecen ajonjolí molido con agua, un remedio que también le suministran a las cabras cuando tienen el mismo problema (18).

La mujer Wayúu guarda una cuarentena que puede extenderse hasta dos meses. En este periodo se baña normalmente todo el cuerpo, pero no la cabeza y se cuida de no "serenarse». Las mujeres primerizas no realizan todos los trabajos caseros, como lavar y cocinar; estas labores son realizadas generalmente por la madre o por una hermana mayor. Si el padre del recién nacido no asume la responsabilidad de sustentar económicamente a la mujer durante el posparto, los tíos maternos son los encargados de esta función (14-19).

Entre los años 2010 y 2012, Nájera \& Tuesca realizaron un estudio en el que analizaron los casos de muerte materna ocurridos en el Departamento de la Guajira (21). El objetivo de su estudio fue identificar si las desigualdades sociales y las características sociodemográficas del departamento incidían de manera negativa en la mortalidad materna. Estos autores encontraron que, para el periodo estudiado, se habían registrado 54 muertes maternas, de las cuales el $61,1 \%$ eran indígenas y el $33,3 \%$ no contaba con ningún estudio. Este estudio concluyó que la tasa de mortalidad materna fue significativamente más elevada en las mujeres indígenas que en las no indígenas.

La presente investigación tiene como objetivo describir el comportamiento de la mortalidad materna de la gestante Wayúu en el municipio de Uribia, Departamento de la Guajira, Colombia, en el periodo comprendido entre el 1 enero de 2010 y 31 diciembre de 2016. La información para esta descripción fue recolectada a partir de los registros históricos sobre el número de nacimientos y defunciones fetales y no fetales reportados y validados por el Departamento Administrativo Nacional de Estadísticas del año 2016 (DANE- 2016)².

Al describir las prácticas de cuidado cultural en las gestantes indígenas Wayúu, se busca que el profesional de enfermería conozca cómo estas prácticas influyen en los indicadores de mortalidad materna. Este conocimiento ha sido poco explorado, por lo que que se hace necesario abordarlo para tomar decisiones y emprender acciones orientadas al cuidado de las gestantes en comunidades indígenas. En consecuencia, la investigación presente ofrece un aporte significativo a la disciplina de Enfermería.

\section{METODOLOGÍA}

Esta investigación corresponde a un estudio descriptivo retrospectivo cuyo objetivo consistió en describir el comportamiento de la mortalidad materna en la población indicada, a partir de información extraída de registros históricos del Departamento Administrativo Nacional de Estadísticas en relación con los casos de muerte materna reportados en el periodo comprendido entre 2010 y 2016. Para realizar esta investigación no se requirió consentimiento informado, dado que los datos recolectados se extrajeron de fuentes documentales. Sin embargo, se realizó una solicitud formal a los entes gubernamentales del Departamento de la Guajira y del municipio de Uribia (Secretaría de Salud Departamental y Municipal) con el fin de obtener la información requerida para este estudio. Los datos obtenidos fueron transcritos a una base de datos elaborada en Microsoft Excel versión 3.5 diseñada especialmente para la investigación. Los datos suministrados no fueron alterados en ningún momento del proceso, de modo que es posible garantizar la veracidad de la información aquí reunida.

Con respecto a los materiales y métodos, en la primera fase de este estudio se revisó la literatura existente en relación con el comportamiento de la mortalidad materna, especialmente en comunidades indígenas. Se hizo énfasis en la búsqueda de factores relacionados con este tipo de evento, tales como: pertenencia étnica, grupo etario, profesional de salud que realiza la atención del parto, causas asociadas a la mortalidad materna y prácticas culturales de cuidado en las gestantes de comunidades indígenas.

La segunda fase consistió en contrastar los hallazgos reportados en el proceso investigativo a la luz de la literatura disponible sobre el fenómeno estudiado. Es preciso aclarar que en el contexto colombiano existen pocos estudios relacionados con la mortalidad materna en la comunidad indígena Wayúu, por lo que la discusión de los resultados en torno a este tópico se realizó con base en los perfiles epidemiológicos y el comportamiento de la mortalidad materna en la distintas regiones del país, de acuerdo con los reportes oficiales del DANE del año 2016

2 El DANE consolida, valida y procesa la información de los nacimientos a partir de los Certificados de Nacido Vivo diligenciados por personal de salud autorizado y funcionarios de Registro Civil. La información de defunciones fetales y no fetales corresponde a la consolidación, codificación de causas de muerte, validación y procesamiento realizado por el DANE, a partir de los certificados de defunción. 
A pesar de los esfuerzos gubernamentales por mejorar la cobertura en el programa de control prenatal, incrementar la atención del parto institucional y desarrollar programas comunitarios en salud con enfoque diferencial étnico, la mortalidad materna sigue cobrando un número importante entre las mujeres de la etnia Wayúu del Departamento de la Guajira. La tendencia de la mortalidad materna en el municipio de Uribia, en específico, mostró un ascenso vertiginoso los primeros cuatro años del periodo estudiado (2010 -2014). El pico de mortalidad materna más alto reportado por el ente territorial del municipio de Uribia ocurrió en el año 2013: se presentaron 7 muertes maternas por 1525 nacidos vivos y todas eran mujeres de la etnia Wayúu. El mayor número de nacimientos ocurridos en el municipio de Uribia durante los periodos estudiados se presentó en los años 2014 y 2015; los recién nacidos de sexo masculino representaron la mayor proporción reportada, $32 \%$ vs $30 \%$.

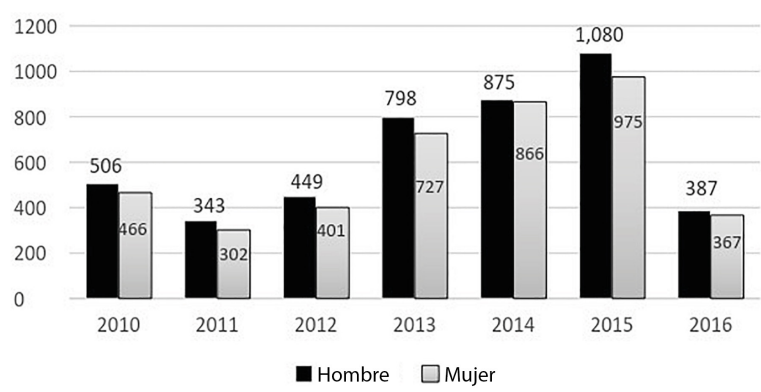

Fuente: Departamento Administrativo Nacional de Estadísticas DANE, Registro de nacimientos por sexo y municipio de residencia. Periodo 2010- 2016.

Gráfico 1. Número de nacimientos por sexo en el municipio de Uribia, Departamento de la Guajira. Enero 2010-Diciembre 2016.

Al determinar el número de nacimientos por área de residencia de la madre, se observa que en la cabecera municipal ocurre el mayor número de nacimientos registrados (5086 Nacimientos). Sin embargo, llama la atención que en las zonas rurales dispersas se ha concentrado un importante número de nacimientos a través de los años (1976 Nacimientos).

Al comparar el número de nacimientos por edad de la madre y sitio de residencia, se puede apreciar que las mujeres entre los 15 y 35 años reportan el mayor número de nacimientos. En este grupo se concentra el mayor número de mujeres embarazadas en el municipio de Uribia, de acuerdo a cifras oficiales del Boletín epidemiológico de la semana 52 del 2016 en Colombia. La mayor razón de mortalidad materna se registró en el área rural con 78,8 muertes maternas tempranas por 100000 nacidos vivos; el $63,7 \%$ se registró en el régimen subsidiado y la mayor razón se presentó en la población de mujeres no afiliadas, con 62 muertes maternas tempranas por 100000 nacidos vivos.

La razón de mortalidad materna en Colombia para mujeres de pertenencia étnica indígena fue de 316,5 muertes maternas tempranas por 100000 nacidos vivos; de estas, el 24,6\% fueron en las mujeres de 20 a 24 años. De otro lado, el número de nacimientos de madres entre 15 y 19 años en el municipio de Uribia ha mostrado una tendencia creciente. Con esto se evidencia un aumento significativo en las tasas de embarazo adolescente, factor que contribuye a la mortalidad materna y perinatal del municipio.

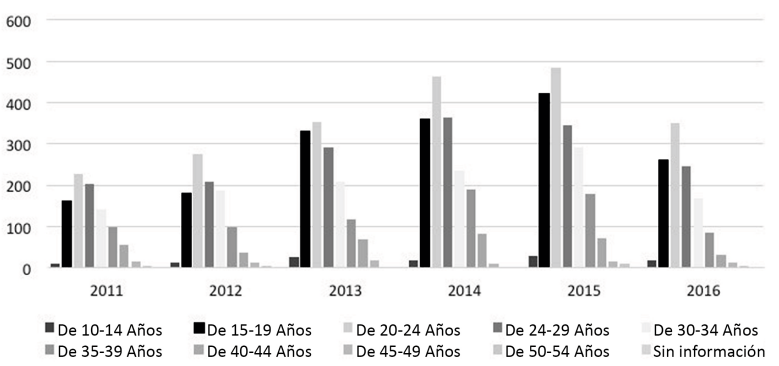

Fuente: Departamento Administrativo Nacional de Estadísticas DANE, Registro de nacimientos por grupo de edad de la madre. Periodo 2010- 2016

Gráfico 2. Número de nacimientos por grupo de edad de la madre en el municipio de Uribia, Departamento de la Guajira. Enero 2010Diciembre 2016.

El municipio de Uribia no es entonces ajeno a realidad nacional; las características demográficas de la población objeto de estudio guardan relación con el panorama nacional. No obstante, la desventaja social que tiene la gestante Wayúu es mucho más evidente, no sólo por las barreras de acceso a los servicios de salud sino también por las barreras geográficas a las que se encuentra expuesta esta comunidad.

Un estudio realizado durante el 2000-2003 por la Organización Panamericana de la Salud sobre mortalidad materna evidenció una serie de desventajas de la población indígena con respecto a la no indígena. Entre las desventajas se destaca el embarazo a edades tempranas, la falta de asistencia médica, el domicilio en zonas rurales dispersas y la baja o nula escolaridad (22). La mayor proporción de muertes maternas ocurre en mujeres indígenas con bajo o ningún nivel educativo. El riesgo de muerte es 10 veces mayor respecto a las no indígenas; en mujeres indígenas de 10 a 14 años el riesgo es cuatro veces mayor, además de pertenecer al régimen subsidiado y haber tenido el parto en el domicilio (23). 
En el Departamento de la Guajira, la atención del parto institucional ha venido aumentando a través de los años. Los esfuerzos por mantener una cobertura útil en la atención del parto institucional se reflejan en los datos estadísticos. Al determinar el número de partos de acuerdo a la persona que realizó la atención del mismo, se puede apreciar que, a medida que crece el número de partos atendidos por personal médico, decrece el número de partos atendidos por parteras. (Gráfico 3)

Para acceder a los servicios de salud institucionales, sin embargo, generalmente la gestante o la puérpera Wayúu tiene que recorrer largas distancias hasta un centro de salud. Las vías y los medios para movilizarse se encuentran restringidos para muchas comunidades indígenas de la etnia Wayúu. Por tanto, existe un grupo significativo de mujeres Wayúu del municipio de Uribia que prefiere la atención del parto en su sitio de asentamiento. Además de las dificultades de acceso a los servicios de salud, las particularidades geográficas y las largas distancias que habría que recorrer, es común que estas mujeres prefieran ser atendidas por una partera de su propia comunidad, por razones culturales.

La atención domiciliaria del parto puede ser un factor desencadenante de complicaciones obstétricas que, si no son atendi-

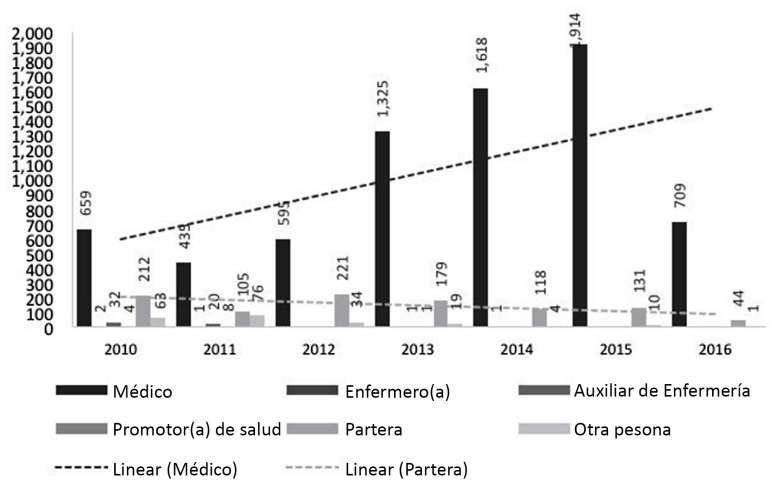

Fuente: Departamento Administrativo Nacional de Estadísticas DANE. Registro del nacimientos por tipo de persona que atendió el parto según el municipio y sitio de ocurrencia. Periodo 1 de enero de 2010 - 31 de diciembre de 2016.

Gráfico 3. Número de nacimientos atendidos por tipo de persona que atendió el parto en el municipio de Uribia, Departamento de la Guajira. 1 de enero 2010-31 de diciembre 2016. das adecuadamente, pueden conducir a la muerte de la madre. La hemorragia posparto (HPP) fue la principal causa de mortalidad materna en el municipio de Uribia. En segundo orden aparecen los trastornos hipertensivos de la gestación. (Gráfico 4)

Las causas de muerte materna por hemorragia, sepsis e hipertensión son dos veces mayor en población indígena que no indígena. Teniendo en cuenta las zonas rurales dispersas, en las que se presentan complicaciones relacionadas con el tiempo prolongado del trabajo de parto (más de 24 horas), también hay riesgos de ruptura de útero, hemorragias severas y retención de la placenta (24-26).

Las causas de mortalidad materna siguen siendo las mismas descritas por la literatura. Las causas más comunes y frecuentes son los trastornos hipertensivos, la hemorragia post parto y la sepsis obstétrica. Sin embargo, para la gestante Wayúu existen complicaciones adicionales en relación con el manejo oportuno de este tipo de condiciones. Adicionalmente, la situación se ve afectada en primer lugar por la remisión oportuna de la mujer a los centros asistenciales y en segundo lugar por la capacidad resolutiva de los prestadores de atención en salud para responder a este tipo de eventualidades.

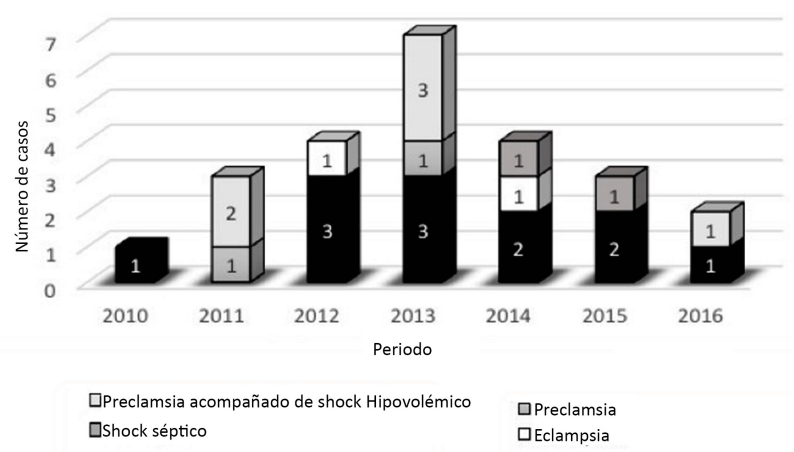

Fuente: Departamento Administrativo Nacional de Estadísticas DANE. Registro de Defunciones no Fetales según el municipio y sitio de ocurrencia. Periodo 1 de enero de 2010 - 31 de diciembre de 2016.

Gráfico 4. Causas de defunción materna en el municipio de Uribia, Departamento de la Guajira. 1 de enero 2010 - 31 de diciembre 2016 


\section{DISCUSIÓN}

Como se mencionó, el municipio de Uribia es habitado en su mayoría por población Wayúu, el $93 \%$ habita en el área rural y el $7 \%$ restante en el área urbana $(1-5,10-12,27)$. Con todo, cada vez son más las mujeres del municipio de Uribia que se acercan a la cabecera municipal para recibir la atención del parto en las instituciones de salud. El mayor número de nacimientos en la cabecera municipal reportado en los periodos estudiados se presentó en el año 2015. Sin embargo, por ser el municipio de Uribia un territorio inhóspito, desértico, semiárido y poco accesible, la mayoría de las indígenas Wayúu prefieren aún permanecer en su lugar de origen y disponer de los recursos locales para dar respuesta a sus necesidades de salud $(27,28)$.

A partir de esta situación es pertinente preguntar qué condiciones comparten los territorios de la Guajira para que se presente una tendencia creciente en el comportamiento de la mortalidad materna. Tres elementos, en efecto, se encontraron como los patrones comunes que juegan un papel decisivo en el comportamiento y la tendencia de este indicador: la pobreza, la etnicidad y la ruralidad. Estos hallazgos ponen de manifiesto la necesidad de desarrollar acciones que contribuyan a superar las brechas, con el fin de adecuar los programas de salud materna a las particularidades de cada pueblo.

En relación con las prácticas de cuidado en las gestantes de comunidades indígenas, Ramos Lanfot realizó una investigación de abordaje cualitativo, con el enfoque de etnoenfermería, propuesto en 1991 por Leininger, en una comunidad indígena Zenú, o Sinú, del departamento de Córdoba $(29,30)$. Los resultados de este estudio describieron cuatro dominios culturales relacionados con el proceso de embarazo: el significado del embarazo para la mujer zenú, las maneras de cuidarse, la comadrona como elemento fundamental en el control del embarazo y la atención de la mujer zenú durante el embarazo y el parto. Este estudio concluyó que las prácticas culturales identificadas en las gestantes indígenas zenú evidencian el apego que tienen hacia sus costumbres y tradiciones y resaltan la confianza que manifiestan respecto a los cuidados y la atención que le brindan las comadronas o parteras de la comunidad. A pesar de lo señalado, el estudio propuesto por Ramos no menciona cuál ha sido la contribución de la comadrona en los indicadores de morbi-mortalidad materna de las gestantes del resguardo indígena Zenú del departamento de Córdoba.

Otros autores, como Oviedo $\mathrm{M}$, et al. realizaron un estudio cualitativo con el objetivo de describir las prácticas de cuidado durante el proceso de gestación en las comunidades Embera y Wuanán, del departamento del Chocó (31). Los datos obtenidos en la investigación reflejan cuatro categorías que dan cuenta de las prácticas de cuidado alrededor de la gestación en las comunidades indígenas estudiadas: los cuidados de la pareja, los cuidados ofrecidos por la familia, la alimentación durante la gestación y las actividades realizadas por la gestante. La decisión de buscar ayuda para la gestante es tomada por el hombre, él es quien determina cuándo y a quién solicitarla. En estas comunidades no se acostumbra a realizar controles periódicos de salud durante el embarazo; sin embargo, se reconocieron dos momentos en los cuales acuden: cuando consideran que la gestante está enferma y cuando se acerca el momento del parto. La partera es quien identifica los problemas que puedan surgir durante el embarazo: "La partera sabe si el niño viene normal, o no, y le avisa al gobernador de la comunidad indígena para que hable con el hombre, para que la lleve al médico" (31). La decisión final de llevarla, en todo caso, sigue siendo potestad del hombre.

Aunque las mujeres reciben apoyo de su comunidad y su familia durante el embarazo, continúan realizando actividades de la vida cotidiana y asumiendo todas las labores del hogar. Se señaló también que los conocimientos y las prácticas de cuidado de las gestantes obedecen a la información que han recibido de sus progenitoras, de manera intergeneracional, y estos elementos son convertidos así en normas culturales (31).

Medina \& Mayca realizaron un estudio etnográfico en comunidades indígenas de la Amazonía peruana, en los pueblos Awajun y Wampis. Con este estudio identificaron que el nacimiento está asociado a componentes de orden cultural que le dan un sentido mágico: el contexto familiar, la administración de infusiones calientes a base de plantas medicinales, el lugar del parto, la participación de la partera, los elementos que serán usados y las dietas, entre otros (32).

En la mayoría de casos de mortalidad materna se identifican factores comunes, como las prácticas culturales en torno al embarazo, parto y posparto, la responsabilidad de la familia en la toma de decisiones cuando existe algún tipo de complicación y las intervenciones realizadas por las parteras o comadronas de la comunidad $(29,32,34-36)$. Sin embargo, poco se conoce sobre el impacto que tienen estas prácticas de cuidado en la reducción de la mortalidad materna y perinatal de las comunidades indígenas $(32,33,37-39)$.

En este sentido, la OMS recomienda articular los diferentes sistemas médicos tradicionales con el sistema oficial de salud a fin de reducir la mortalidad, morbilidad y discapacidad en poblaciones pobres y marginadas (40). Es importante señalar que, para que esta recomendación sea implementada, se debe partir del respeto por las diferentes culturas $(33,37,38)$. Los grupos étnicos poseen maneras propias de entender lo que les rodea y tienen fundamentadas razones para hacerlo; por esto, es necesario que el sistema de salud reconozca y respete dichas prácticas y razones y, a partir de ellas, genere espacios para la construcción y articulación de acciones encaminadas a mejorar la salud de las poblaciones indígenas (41-43).

Tomando en consideración esta premisa, la inclusión de un enfoque diferencial se convierte en una de las alternativas claves para el fortalecimiento de la salud materna y perinatal de esta población. La apuesta para lograrlo tiene como base la construcción de relaciones de confianza entre las autoridades tradicionales y la institucionalidad. 


\section{CONCLUSIONES}

Por razones que atienden a la dificultad de acceder a los servicios de salud, a las características geográficas de la región o a las prácticas tradicionales de la comunidad, hay un número significativo de mujeres de la comunidad indígena Wayúu del municipio de Uribia que prefieren llevar a cabo los partos en su sitio de asentamiento. Aunque esta situación ha disminuido con el tiempo, gracias a los esfuerzos por mejorar la salud materna en el departamento de la Guajira, la atención domiciliaria del parto persiste y es uno de los factores más asociados con la alta tasa de mortalidad materna. Este factor es una evidencia, a su vez, de la vulnerabilidad social y de salud a la que están expuestas estas comunidades.

La atención de partos domiciliarios, por parteras o familiares, es una práctica cultural habitual de poblaciones indígenas como la Wayúu, pero puede ser la causa de complicaciones obstétricas como la hemorragia posparto, identificada como la principal causa de muerte materna. Es necesario, entonces, articular acciones que no desconozcan las prácticas y saberes propios de las comunidades indígenas, ni sus necesidades en temas de salud.

Finalmente, aunque no se trabajó directamente con las comunidades indígenas Wayúu, es recomendable, para un siguiente estudio en relación con la población Wayúu u otro grupo étnico, poder interactuar con las comunidades y proponer estudios etnográficos o Investigación de Acción Participativa IAP, con el fin de determinar cómo influyen las prácticas de cuidado cultural durante la gestación en los indicadores de mortalidad materna y perinatal del pueblo Wayúu.

Cómo citar este artículo: Alonso Bula Romero, Galarza, K. Mortalidad materna en la gestante Wayúu de Uribia, Departamento de la Guajira, Colombia. Estudio descriptivo año 2016. Enfermeria (Montev.). [Internet]. 2017 Jun [citado xxxxx]; 6 (1) 46-53. Disponible en: http://dx.doi.org/10.22235/ech. v6i1.1369

\section{REFERENCIAS BIBLIOGRÁFICAS}

1. Hernández Romero, A. La visibilización estadística de los grupos étnicos colombianos. DANE. Bogotá: Imprenta Nacional. [citado May 2017]. Disponible en https:// www.dane.gov.co/files/censo2005/etnia/sys/visibilidad_ estadistica_etnicos.pdf

2. Departamento de la Guajira. Análisis de situación de salud, municipio de Uribia, la Guajira 2016. Secretaría Departamental de Salud- Gobernación de la Guajira.

3. Departamento de la Guajira.Análisis de situación de salud, municipio de Uribia la Guajira 2014 Secretaría Departamental de Salud- Gobernación de la Guajira.

4. Departamento de la Guajira.Análisis de situación de salud, municipio de Uribia la Guajira 2012 Secretaría Departamental de Salud- Gobernación de la Guajira.
5. Departamento de la Guajira.Análisis de situación de salud, municipio de Uribia la Guajira 2010 Secretaría Departamental de Salud- Gobernación de la Guajira.

6. Boletín Epidemiológico Semanal. Dirección de Vigilancia y Análisis del Riesgo en Salud Pública. Semana epidemiológica número 52 de 2014 (Consultado 27 dic. al 02 ene.)

7. Boletín Epidemiológico Semanal. Dirección de Vigilancia y Análisis del Riesgo en Salud Pública. Semana epidemiológica número 52 de 2013 (27 dic. al 02 ene.)

8. Comisión Económica para América Latina y el Caribe. Salud materno-infantil de pueblos indígenas y afrodescendientes de América Latina: aportes para una relectura desde el derecho a la integridad cultural. [Internet] Chile: CEPAL; 2010. [Citado 11 de enero de 2016]. Disponible en: http:// www.eclac.org/cgi-bin/get-Prod.asp?xml=/publicaciones/ $\mathrm{xml} / 9 / 41579 / \mathrm{P} 41579 . x \mathrm{xl} \& \mathrm{xs}=/$ celade/tpl/p9f.xsl\&base= /celade/tpl/top-bottom.xsl

9. Ortega J. Géneros y generaciones: conducta reproductiva de los Mayas de Yucatán, México. Salud Colectiva. [Internet]. 2015 [citado 10 de enero de 2016]; 2 (1): 75-89. Disponible en: http://www.scielo.org.ar/scielo.php?scrip$\mathrm{t}=\mathrm{sci}$ _arttext\&pid=S185182652006000100007\&lng=es\&n$\mathrm{rm}=\mathrm{iso}$.

10. Departamento de la Guajira. Análisis de situación de salud, municipio de Uribia la Guajira 2013 Secretaria Departamental de Salud- Gobernación de la Guajira.

11. Departamento de la Guajira. Análisis de situación de salud, municipio de Uribia la Guajira 2011 Secretaría Departamental de Salud- Gobernación de la Guajira.

12. Departamento de la Guajira .Análisis de situación de salud, municipio de Uribia la Guajira 2015 Secretaría Departamental de Salud- Gobernación de la Guajira.

13. Informe Final MIRA: Alta Guajira - Uribia (La Guajira), Colombia. Desabastecimiento de alimentos en comunidades Wayúu de la Alta Guajira 2014. [Internet]. [citado May 2017]. Disponible en: https://www.humanitarianresponse.info/es/operations/colombia/assessment/informe-final-mira-municipio-de-uribia-desabastecimiento-de-alimentos

14. Bodnar Y. Pueblos indígenas de Colombia: Apuntes sobre la diversidad cultural y la información Sociodemográfica. Trabajo de grado. Bogotá D.C. Universidad Externado de Colombia. Facultad de Sociología. 2006. 231-262 p.

15. Unidad de Planeación Minero Energética. Los Wayuu. Ciclo de vida. [Internet] [citado Nov 2015]. Disponible en: http://www.upme.gov.co/guia_ambiental/carbon/areas/ minorias/contenid/wayuu.htm

16. Aniyar, A. La concepción del mundo y la vida guajiro. En Revista de la Facultad de Derecho, 14 (43): 391-429.

17. Boletín Epidemiológico Semanal. Dirección de Vigilancia y Análisis del Riesgo en Salud Pública. Semana epidemiológica número 52 de 2012 (27 dic. al 02 ene.)

18. Colmenares, F. Acerca del blanqueo omajayuraaa entre los guajiros. En Venezuela Misionera, XXXII (371): 58-59.

19. De Cora M. Kuai-Mare. Mitos aborígenes de Venezuela. Caracas: Herrero Hermanos; 1972 
20. López Cuesta L. Elaboración participativa del plan integral de vida del resguardo de lomamato - guajira. Revista de la unidad de educación de la facultad de ciencias humanas y sociales horizontes pedagógicos. 2012; 14 (1); 68-96.

21. Nájera Arregocés $S$, Tuesca Molina R. Desigualdades y características sociodemográficas de la mortalidad materna en La Guajira, Colombia, 2010-2012. Rev Panam Salud Pública. Apr. /May. 2015; 37 (4-5)

22. Organización Panamericana de la Salud - OPS/OMS. Reducción de la mortalidad Materna y Neonatal, con enfoque intercultural, de derechos y de género. Estrategia de cooperación interagencial en salud materna y neonatal indígena 2015-2019.

23. Instituto de Salud Pública. Pontificia Universidad Javeriana. Determinantes sociales de las desigualdades en mortalidad materna y neonatal en las comunidades indígena arhuaca y wayuu: Evidencias y propuestas de intervención. Documento técnico. Bogotá: PUJ; 2016.

24. Valdebenito et al. Salud intercultural: Impacto en la identidad social de mujeres aimaras. Acta bioeth. [Internet]. 2006 [citado 13 de Enero de 2016]; 12 (2): 185-191. Disponible en: http://www.scielo.cl/scielo.php?script=sci_arttext\&pid=S1726-569X2006000200007\&lng=es

25. Umeora OUJ, Egwuatu VE. The role of unorthodox and traditional birth care in maternal mortality. Tropical Doctor. 2010; 40(1): 13-17

26. Organización Mundial de la Salud. Disponible en: http:// www.who.int/mediacentre/factsheets/fs348/es/Consultado el 06 de mayo de 2017.

27. Boletín Epidemiológico Semanal. Dirección de Vigilancia y Análisis del Riesgo en Salud Pública. Semana epidemiológica número 52 de 2016 (27 dic. al 02 ene.)

28. Boletín Epidemiológico Semanal. Dirección de Vigilancia y Análisis del Riesgo en Salud Pública. Semana epidemiológica número 52 de 2015 (27 dic. al 02 ene.)

29. Ramos Claudia P. Prácticas culturales de cuidado de gestantes indígenas que viven en el Resguardo Zenú ubicado en la Sabana de Córdoba. [Tesis de Maestría] Bogotá: Universidad Nacional de Colombia; 2011. Disponible en: http:// www.bdigital.unal.edu.co/5416/1/539507.2011. pdf.

30. Leininger Madeleine. Transcultural Nursing Concepts. Theories, Research \& practices Second Chapter 3. Overview of Leininger's Culture Theory. New York McGraw- Hill, Inc College Custom Series; 1995. 3-52.

31. Oviedo $\mathrm{M}$, et al. Comunidades Embera y Wounaan del departamento de Chocó: prácticas de cuidado durante el proceso de gestación. Rev. Fac. Nac. Salud Pública 2014; 32(2): 17-25.

32. Medina A, Mayca J. Creencias y costumbres relacionadas con el embarazo, parto y puerperio en comunidades nativas Awajun y Wampis. Rev. Perú. Med. Exp. Salud pública [Internet]. 2006 ene-mar [citado 22 enero 2016]; 23 (1). Disponible en: http://www.scielo.org.pe/scielo.php?scrip$\mathrm{t}=\mathrm{sci}$
33. Saenz T. Prácticas indígenas y tradicionales respecto al embarazo, el parto, el amamantamiento y la nutrición. Lima: Asociación Urpichallay; 2002. Disponible en: http:// www.lactared.com.pe/conferencia/ igam2002/resúmenes/ PRACTICAS\%20INDIGENAS\% OY\%20TRADICIONALES-TULA \%20SAENZ.doc.

34. Bello Á, Rangel M. La equidad y la exclusión de los pueblos indígenas y afro descendientes de América Latina y el Caribe, Santiago de Chile [Internet]. [22 01 2016], Disponible en:(www.cholonautas.edu.co pe/modulo/aproad/ equidad\%20exclusion\%20\%Bello-\%rangel.pdf)

35. Organización Mundial de la Salud. Parteras tradicionales: Guía práctica para el adiestramiento, la evaluación y la articulación de este personal en los servicios de salud. Ginebra: OMS; 1979.

36. Vargas R, Naccarato P. Allá las antiguas abuelas eran parteras. Etnografía de las parteras empíricas. Lima: Centro de la Mujer Peruana Flora Tristán; 1995.

37. Myrna C. Etnia, Cultura y salud: La experiencia de la salud intercultural como una herramienta para la equidad en las regiones autónomas de Nicaragua. Washington DC: Organización Panamericana de la salud.

38. Cárdenas $\mathrm{C}$. Conocimientos y prácticas médicas tradicionales de la población colona asentada en la carretera Puerto Maldonado - Mazuko. Amazonía Perú. 1999; 26: 157-210.

39. Chávez et al. Rescatando el autocuidado de la salud durante el embarazo, el parto y al recién nacido: representaciones sociales de mujeres de una comunidad nativa en Perú Texto \& Contexto Enfermagem. [Internet]. [Citado 22 enero 2016]. Disponible en: www.redalyc.uaemex.mx/redalyc/src/inicio/ArtPdfRed.jsp?iCve-=71416412

40. Organización Panamericana de la Salud, Organización Mundial de la Salud. Prestación de servicios de salud en zonas con pueblos indígenas. [Internet]. [Citado 5 de enero de 2016]. Disponible en: http://new.paho.org/ecu/ index.php?option=com_content\&task=blogcategory\&i$\mathrm{d}=684$ \& $\mid$ temid $=25$

41. Muñoz et al. Interculturalidad y percepciones en salud materno-perinatal, Toribio Cauca 2008-2009. Rev. Univ. Ind. Santander. Salud [Internet]. 2012 abril [citado 19 de junio de 2013]; 44 (1): 39-44. Disponible en: http:// www.scielo.org.co/scielo.php?script=sci_arttext\&pi$d=S 0121-08072012000100005 \&$ Ing.

42. Mayca J, et al. Percepciones del personal de salud y la comunidad sobre la adecuación cultural de los servicios materno perinatales en zonas rurales andinas y amazónicas de la región huánuco. Rev Perú Med Exp Salud Pública. [Internet] 2009 [citado 19 de enero de 2016]; 26 (2): 145-60. Disponible en: http://www.scielo.org. pe/pdf/rins/v26n2/ a04v26n2.pdf

43. Castro R, Bronfman M, Loya M. Embarazo y parto entre la tradición y la modernidad: el caso de Ocuituco. Estudios Sociológicos. [Internet] 1991 sep-dic [citado 26 de Enero de 2016]; 9 (27). Disponible en: http://www.jstor.org/discover/10.23 
